\title{
Fused Deposition Modeling 3D Printing Technology in Textile and Fashion Industry: Materials and Innovation
}

\author{
Samit Chakraborty ${ }^{1}$ and Manik Chandra Biswas ${ }^{2 *}$ \\ ${ }^{1}$ Textile Technology Management, Department of Textile and Apparel, Technology Management, North Carolina State University, USA \\ ${ }^{2}$ Fiber and Polymer Science, Department of Textile Engineering, Chemistry and Science, North Carolina State University, USA
}

*Corresponding author: Manik Chandra Biswas, Fiber and Polymer Science, Department of Textile Engineering, Chemistry and Science, Wilson College of Textiles, North Carolina State University, USA.

Received Date: November 05, 2019

Published Date: November 13, 2019

\begin{abstract}
Over the last ten years, researchers, technologists, designers, retailers and manufacturers have worked on embedding 3D printing (3DP) technology in all types of manufacturing industries including textile, apparel and fashion industry. The development of this technology has enabled designers to design more conveniently and manufacturers to reduce production time and cost significantly. Although 3D printing technology is gradually developing in textile and fashion industry, textile and fashion researchers and manufacturers have mostly worked on designing wearable technology, footwear and fashion accessories but rarely on everyday wearing. This review paper aims to describe the Fused Deposition Modeling (FDM) 3D printing technology, as it is mostly used by textile and fashion, designers, manufacturers and researchers for developing various textile patterns and fashion items.
\end{abstract}

Keywords: 3D printing; Textile fashion, Waste management; Sustainable fashion; Circular textile

\section{Introduction}

\section{A brief overview of 3D printing technology}

3DP is a computer assisted design (CAD)/computer assisted manufacturing (CAM) technology where a component or the whole substance is manufactured in a layer-by-layer fashion based on a 3D digital model utilizing liquid or rigid materials [13]. Using exact proportion of materials to produce each layer and trimming the unneeded materials to get the required shape makes this process more sustainable and cost effective compared to conventional production process [4]. There are two major phases involved in 3D printing pre-processing and post processing [5]. Vanderploeg, Lee, \& Mamp (2017) presented a general outline of 3D printing production. The pre-process of this production starts with designing the model using required software (e.g. - Rhino, Materialise Magics software, Repetier-Host software, AutoCAD, 3Ds Max etc.) [6]. The design can be altered by adjusting the computational algorithms in order to correct any measurement or add any size parameter for individual customers [4]. The next stage involves dividing the model into horizontal layers and then converting each layer into $\mathrm{x}$ and $\mathrm{y}$ dimensions. "The printer defines the coordinates and print the layers, fusing the material to shape a physical object" [7]. Then this CAD controlled digital file data is transferred to the printer to set up a communication between CAD and CAM. The CAM part of the printer then starts producing the final object based on the design instruction sent to it. The post process phase includes sanding, polishing, further dyeing or painting that are done to enhance the surface finish of the object followed by removing the unwanted print edges $[4,8]$.

Some printers cannot produce whole garment at a time. In such circumstance, multiple parts are printed and then joined together to make the complete garment [8]. Textile and fashion manufacturers generally use the following materials, methods and machines for designing and producing textile fabric, fashion garments, footwear and fashion accessories-

Materials: PET (polyethylene terephthalate) PA (polyamide), ABS (Acrylonitrile butadiene styrene), PLA (Polylactic acid), PVA (Polyvinyl alcohol), metal powder, ceramics and photopolymer 
resin to produce fabric of various textures, long dresses, garment components and functional textiles, footwear and metal accessories $[9,4]$.

Methods: The feasibility of the design and desired outcome largely depend on the selection of the appropriate method or process [2].

1. Binding Process- stereolithography (SLA), selective laser sintering (SLS), inkjet printing [7,4].

2. Deposition Process- fused deposition modeling, and 3D microfiber extrusion [10].

Machines: FDM printer X400, Materialise's Mammoth SL, 3DN-300, Urtidium B200, Objet Connex Stratasys and MakerBot Replicator 2X $[9,4]$.

\section{Research focus on fused deposition modeling (FDM)}

According to researchers not all methods available in the market are applicable and cost effective in producing 3D printed textile and fashion items [11-13]. Therefore, selecting the appropriate and cost effective one is a crucial task for textile and fashion, technologists and manufacturers [2]. Overcoming the technical challenges related to material optimization and operation control are also the critical in 3DP process. SLA, SLS, polyjet and DLP have been applied to a limited extent in textile and fashion industry. On the other hand, FDM is now widely used to develop textile and fashion products because of great availability of materials, simple mechanism and low initial investment $[11,12,6,4,2]$. Therefore, this paper presented an in-depth detail of Fused Deposition Modeling (FDM) technology in developing textile structures and fashion wear.

\section{Fused Deposition Modeling (FDM)}

FDM technology uses wire-shaped and production-grade thermoplastic filament that is heated inside a temperaturecontrolled nozzle connected to a printing head. The nozzle extrudes the molten thermoplastic filament layer by layer onto a print bed above the build platform as per the CAD design model. This platform is heat able and can be lowered according to the selected layer thickness and height each time a layer is printed [12]. This technology allows to produce firm, durable and dimensionally stable products with more impeccability compared to other 3D printing technologies (Stratasys, n.d.). Figure 1 presents a glance of different stages involved in FDM technology-based 3D printing. Figure 2a shows the design development process, Figure $2 \mathrm{~b}$ points supply and extrusion of filament, Figure $2 \mathrm{c}$ respectively shows how extrusion head draws the product and support structure is added. Figure $2 \mathrm{~d}$ shows removal of support. Figure 2e presents the final product. [14].

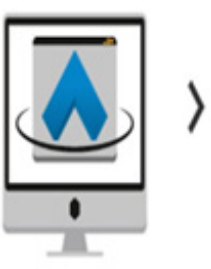

(a)

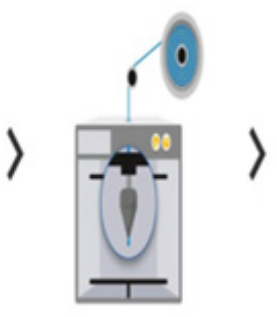

(b)

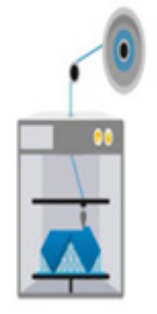

(c)

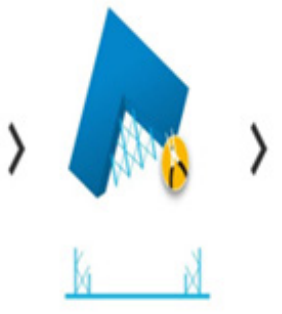

(d)

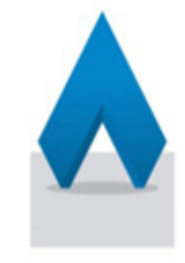

(e)

Figure 1: Different stages involved in FDM based 3D printing [14].

\section{Materials used in FDM}

The filament applied during 3DP processing is manufactured from molten thermoplastic polymer constituted of fillers, fibers, dyes, and other additives [10]. Among the various types of thermoplastics used in FDM, ABS, PLA, polycarbonate (PC), and polyamides (PA) are considered the notable ones [10]. Soft PLA allows printing smooth, shiny and lace-like fabric structures. Since ABS is hard and stiff, its usage provides additional movement to materials and thus makes it ideal for creating joints [15]. PLA and soft PLA are mostly applied because of having mechanical properties better than ABS and PA. Soft PLA prevents the surface from being rough $[10,12,16]$. Figure 2 shows filaments made of ABS, PLA and PC.

Using PLA and soft PLA (mixed with softener) in combination with other materials such as- Ninjaflex, BendLay (butadiene) and thermoplastic elastomer (TPE) produces knitted and woven products, and garment parts with improved physical and mechanical properties. Manufacturers are now also using reinforcing material with PLA, ABS or PC. This type of filament provides more strength and durability compared to synthetic polymer-based filament [17]. Cellulosic fiber is now also incorporated with these enhance the flexibility of final product [18]. Table 1 shows the parameter of PLA filament required for FDM 3D printing $[6,19,15,4]$.

Table 1: Parameter of PLA filament.

\begin{tabular}{|c|c|}
\hline Diameter of the filaments & $1.88-3 \mathrm{~mm}$ \\
\hline Elongation at break & PLA- 4\%, Soft PLA- up to 200\% \\
\hline Young's modulus & PLA- 2600 MPa, Soft PLA -1968 MPa \\
\hline Polymer flow & $100 \%$ \\
\hline
\end{tabular}




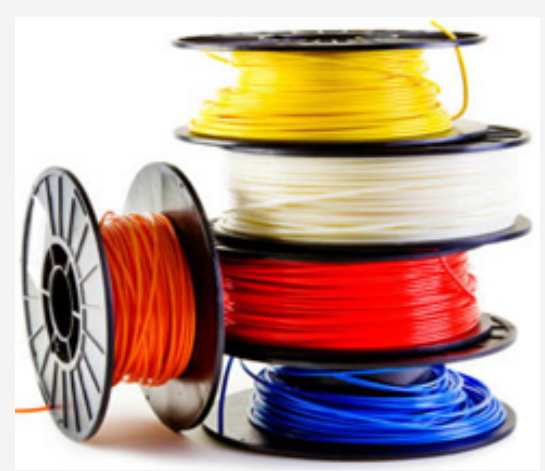

Figure 2: Different types of filament used for FDM based 3D printing [27].

\section{Machine used in FDM}

MakerBot replicator 2 and German RepRap FDM printers are mostly used to design woven, weft knitted, lace-like and net structured fabric or develop 3D print design on cotton, wool, viscose fabric and polyester net $[12,15,16]$. These printers are compatible with both developed closed and open source softwareprogramming options [7]. Depending on the design required, textile and fashion, manufacturers, researchers and designers can use RepRap X400 printer with following parameters (shown in Table 2) for producing desired textile structures and apparel panels $[20,12,16]$.

Table 2: Features of $\mathrm{X} 400$ printer

\begin{tabular}{|c|c|}
\hline Build platform of dimensions & $350 \mathrm{~L} \times 400 \mathrm{~W} \times 310 \mathrm{H} \mathrm{mm}$ \\
\hline Print speed & $10-200 \mathrm{~mm} / \mathrm{s}$ \\
\hline Layer height & $0.2-0.5 \mathrm{~mm}$ \\
\hline $\begin{array}{c}\text { Standard filament/nozzle } \\
\text { diameter }\end{array}$ & $1.75 \mathrm{~mm} / 0.4 \mathrm{~mm}$ \\
\hline Nozzle diameter & $0.25-0.8 \mathrm{~mm}$ \\
\hline Nozzle material & Brass or hardened steel \\
\hline
\end{tabular}

The important aspect about such types of printers is that sometimes they may be supplied in an unassembled package. In such cases, manufacturers or designers may require joining all the components together using open source software [7]. Figure 3 displays an FDM X400 printer and its main parts, manufactured by German manufacturer RepRap company. Figure 4a exhibits the printing head, build platform, extruder nozzle and motors that can be moved into different coordinates.

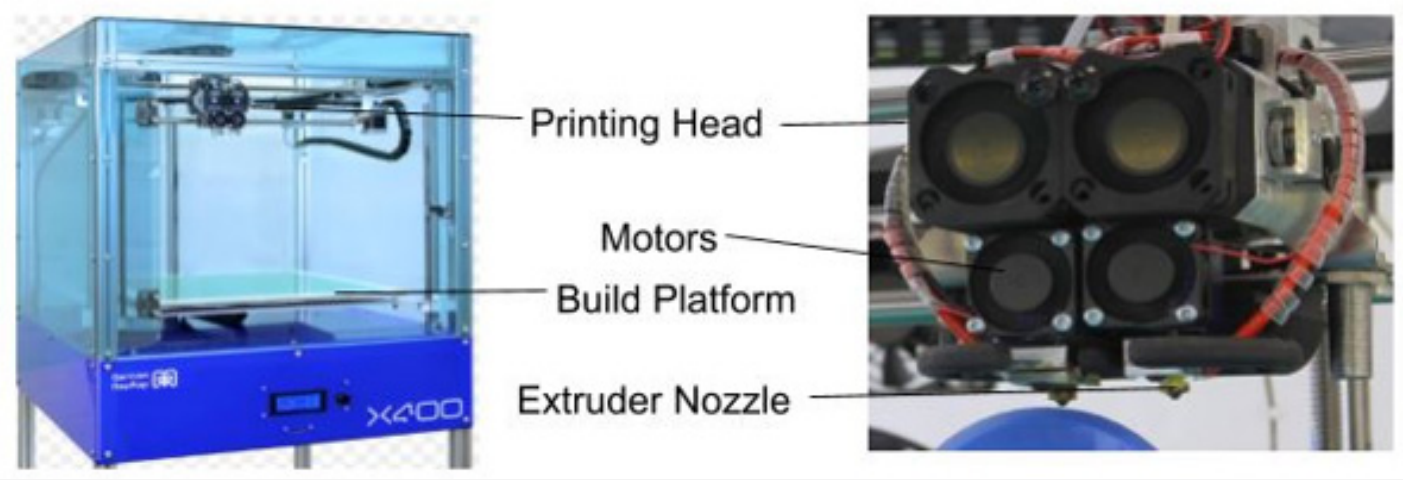

Figure 3: FDM X400 printer [28].

\section{Process description of FDM}

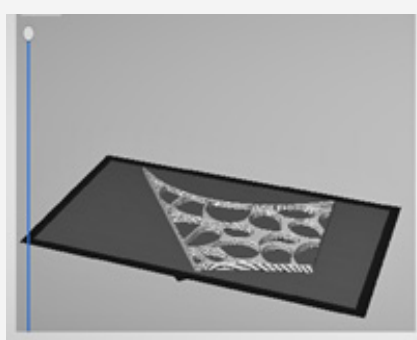

(a)

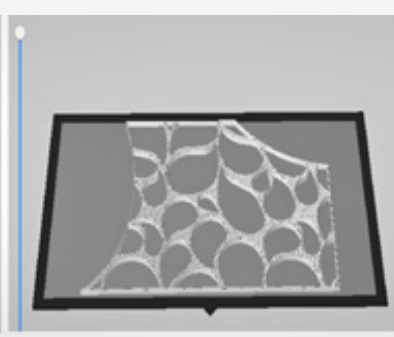

(b)

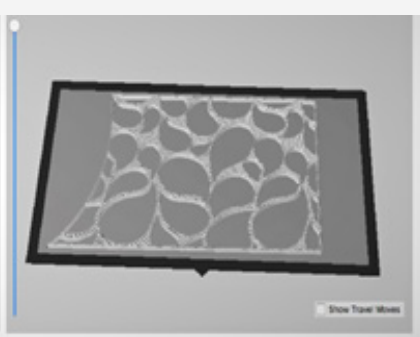

(c)

Figure 4: Front, shoulder and back panels designed in computer [30].

Pre-processing: The preprocessing phase starts with designing the unit cell structure of the material that will be developed, because each cell structure has its own distinctive physical and mechanical properties. For instance, woven fabric structure is known for having structural firmness, nonwoven for fiber material coherence and knit structure for sustaining multi-directional deformation [17]. Generally, Blender software is used for designing the 3D textile structures and utilized netfabb when the model required further modification. Then this modified STL file is opened with repetier host. It slices the model into a layer pattern before sending the final file to the 3D printer $[2,9,15]$. A resolution is set before the print head moves across the build platform [28]. Different processes use different resolutions for printing products. "The resolution describes the layer thickness and outline accuracy of each layer" 
[7]. It is usually defined as spot per unit length and restricted by the nozzle diameter. The higher resolution causes realization of more detailed characteristics realization. Thus, its contribution is crucial in determining the build time [17]. Sun, \& Valtas [30] presented an example computer aided design development in their research, in which they showed how a CAD software designed 3 main parts of a garment- front, shoulder and back parts, as shown Figure 4.

Printing process: The thermoplastic filament used in this method is heated to one degree Celsius above its boiling point before it is dispensed through a nozzle and distributed in layer by layer onto the building platform $[6,4]$. This technique helps the material to harden fast and retain its structural outline almost immediately after being dispersed and bound to the layer under it [4, 2]. While using soft PLA the extruder temperature is maintained around $195^{\circ} \mathrm{C}$ (for Ninjaflex- $220^{\circ} \mathrm{C}, \mathrm{ABS}-230^{\circ} \mathrm{C}, \mathrm{PA}-240^{\circ} \mathrm{C}$ ) and the printing bed temperature around $60-70^{\circ} \mathrm{C}$. The successive layers are printed based on rectilinear infill pattern and at specific angles (generally at $45^{\circ}$ angle and "Z" distance from the bed) $[11,15,12,16]$. Figure 6 shows FDM technology involved 3DP process. The figure shows a build material spool (can be placed either above the printing head or below the build platform) that supplies the filament towards a pair of pinch rollers. Then it is passed through the liquefier head. The nozzle at the end of the head ejects the fluid onto the print bed above the build platform. The support part that temporarily supports the structure [22] (Figure 5).

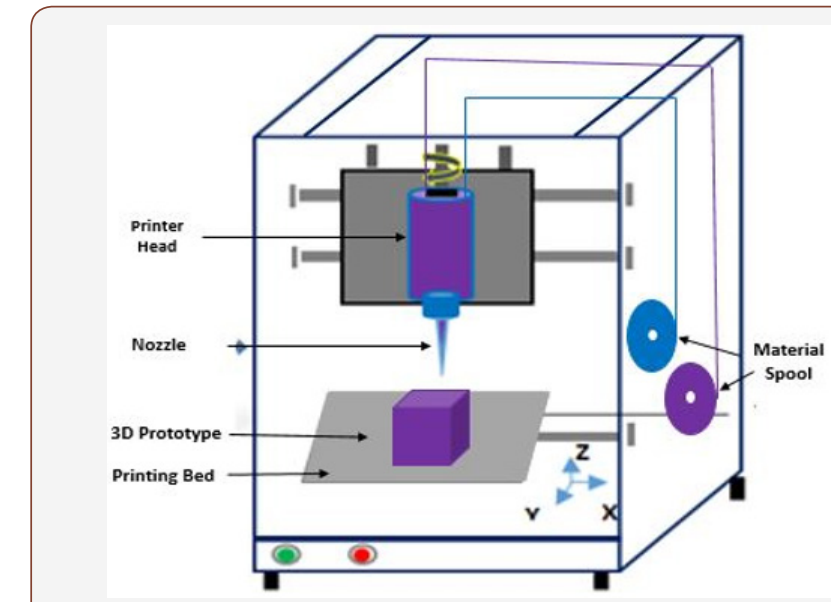

Figure 5: Schematic diagram of FDM 3D printing.

Ligon et al. [10] mentioned that for a filament viscosity of molten filament and its tensile modulus, surface energy, flow property, build speed, and compatibility with the model developed are crucial for a perfect build process. An improper fusion of distributed strands with the adjoining polymer layers can cause a fragile surface structure and significantly affect the mechanical properties of the resulted product. Speed of filament supply, die swelling, molecular orientation, tendency towards crystallinity, processing factors, properties of added dyes or other fillers are maintained during processing, as they contribute to enhance the surface finish, dimensional precision, physical characteristics, mechanical attributes and efficiency of the procedure [6,23-25].

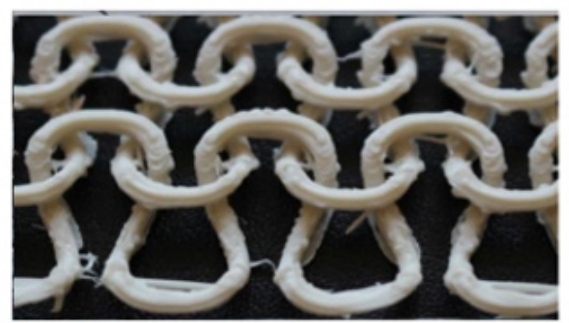

(a)

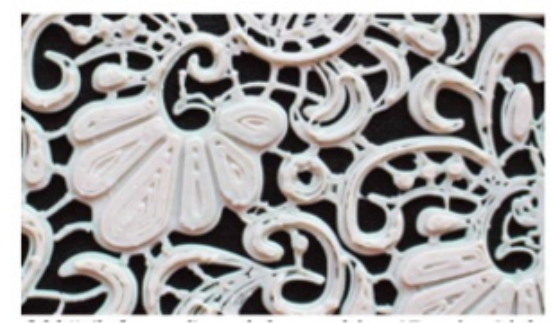

(b)

Figure 6: 3D printed (a) weft knit structure and (b) lace structure [15].

During printing nozzle must be kept very close to the printing bed. The printing bed height is adjusted throughout the process and especially while creating layers $[11,12]$. FDM process requires having support structures, made of either a new added material or the same one as applied during making layers. Hence, based on the software adopted and model created, it is possible to develop braided, weft knitted or woven fabric structures with visible stitches, garment panel, multi-layer multi-color lace-like pattern of different sizes (both loose and reinforced structure) and fabrication of composite [15,12,16]. Melnikova et al. [15] used FDM technology to produce weft knit (Figure 6a) and lace structures (Figure 6b).

Using multi-colored and multifunctional materials such as conductive and insulative fillers with PLA or ABS may result in developing a 3D printed woven composite as well [17].
FDM printer with two or more nozzles also allows printing harder parts, such as eyelets, buttons or other accessories, by facilitating a mixture of multi materials [15]. The parts printed in several pieces are joined or interlocked together, which leads to a more intricate design compared to whole garment manufacturing process. This assembly process varies from style to style [5].

Post-processing: At this stage the support is manually removed without damaging the product. Even after the careful removal of support materials some burrs may be still there with final product and then it is kept in a liquid solution until the remaining support materials completely dissolve into it. Manufacturers should also minimize the staircasing effect $[5,4]$. The aesthetic and adhesion properties of the complete products are then tested. The final products may require drying, brushing, trimming the strands on 
the edge and surface painting. Adding reinforcing plastic or creating grommets alongside the edge of the fabric can prevent it from fraying. The finishing speed during the post-process stage depends on the intricacy required and accuracy of the style desired $[23,9]$.

\section{Conclusion}

The research paper presented in-depth details of fused deposition modeling method including the material and machine used in this technology. It also stated different parameters and factors that should be considered throughout the process. The figures demonstrated in different processes were presented in layout manner to make the fused deposition modelling technology more comprehensive to the readers than earlier. The elaborate discussion on FDM will enable researchers, designers and manufacturers to understand how this technology can be applied in textile, apparel and fashion industry.

\section{Acknowledgement}

This research did not receive any specific grant from funding agencies in the public, commercial, or not-for-profit sectors.

\section{Conflict of Interest}

The authors declare no conflict of interest.

\section{References}

1. Biswas MC, Tiimob BJ, Abdela W, Jeelani S, Rangari VK (2019) Nano silica-carbon-silver ternary hybrid induced antimicrobial composite films for food packaging application. Food Packaging and Shelf Life 19: 104-113.

2. Wang $Q$, Sun J, Yao Q, Ji C, Liu J, et al. (2018) 3D printing with cellulose materials. Cellulose 25(8): 4275-4301.

3. Biswas MC, Jeelani S, Rangari V (2017) Influence of biobased silica/ carbon hybrid nanoparticles on thermal and mechanical properties of biodegradable polymer films. Composites Communications 4: 43-53.

4. Vanderploeg A, Lee SE, Mamp M (2017) The application of 3D printing technology in the fashion industry. International Journal of Fashion Design, Technology and Education 10(2): 170-179.

5. Oropallo W, Piegl L (2015) Ten challenges in 3D printing. Engineering With Computers 32(1): 135-148.

6. Ahn D, Kweon J, Kwon S, Song J, Lee S (2009) Representation of surface roughness in fused deposition modeling. Journal of Materials Processing Technology 209(15-16): 5593-5600.

7. Ghodsi SB (2015) Atossa 3D printed footwear design. Rochester Institute of Technology.

8. Yap YL, Yeong WY (2014) Additive manufacture of fashion and jewellery products: A mini review: This paper provides an insight into the future of 3D printing industries for fashion and jewellery products. Virtual and Physical Prototyping 9(3): 195-201.

9. Taylor A, Unver E (2014) 3D Printing-Media Hype or Manufacturing Reality: Textiles Surface Fashion Product Architecture.

10. Ligon SC, Liska R, Stampfl J, Gurr M, Mülhaupt R (2017) Polymers for 3D printing and customized additive manufacturing. Chem Rev 117 (15): 10212-10290.
11. Grimmelsmann N, Kreuziger M, Korger M, Meissner H, Ehrmann A (2018) Adhesion of 3D printed material on textile substrates. Rapid Prototyping Journal 24(1): 166-170.

12. Korger M, Bergschneider J, Lutz M, Mahltig B, Finsterbusch K, et al. (2016) Possible Applications of 3D Printing Technology on Textile Substrates. IOP Conference Series: Materials Science and Engineering 141(1): 012011.

13. Lopes L, Silva A, Carneiro 0 (2018) Multi-material 3D printing: The relevance of materials affinity on the boundary interface performance. Additive Manufacturing 23: 45-52.

14. Materialise (2018) Fused Deposition Modeling.

15. Melnikova R, Ehrmann A, Finsterbusch K (2014) 3D printing of textilebased structures by Fused Deposition Modelling (FDM) with different polymer materials. IOP Conference Series: Materials Science and Engineering 62(1): 012018.

16. Sabantina L, Kinzel F, Ehrmann A, Finsterbusch K (2015) Combining 3D printed forms with textile structures - mechanical and geometrical properties of multi-material systems. IOP Conference Series: Materials Science and Engineering 87(1): 012005.

17. Quan Z, Wu A, Keefe M, Qin X, Yu J, et al. (2015) Additive manufacturing of multi-directional preforms for composites: opportunities and challenges. Materials Today 18(9): 503-512.

18. Liu W, Zhou J, Ma Y, Wang J, Xu J (2017) Fabrication of PLA Filaments and its Printable Performance. In IOP Conference Series: Materials Science and Engineering 275(1): 012033.

19. Gérard T, Noto T, Budtova T (2012) Tensile Properties of Pla And Phbv Blends: Anomalous Elongation and Aging. In $11^{\text {th }}$ European Symposium on Polymer Blends-Polyblends 2012, Donostia - San Sebastian, Spain.

20. German RepRap GmbH. x400 3D Printer | German RepRap GmbH.

21. Warnier C, Verbruggen D, Ehmann S, Klanten R (2014) Printing things: visions and essentials for 3D printing. Gestalten, Germany.

22. Ning F, Cong W, Hu Y, Wang H (2017) Additive manufacturing of carbon fiber-reinforced plastic composites using fused deposition modeling: Effects of process parameters on tensile properties. Journal of Composite Materials 51(4): 451-462.

23. Jiang K, Gu Y (2004) Controlling Parameters for Polymer Melting and Extrusion in FDM. Key Engineering Materials 259-260: 667-671.

24. Sood A, Ohdar R, Mahapatra S (2010) Parametric appraisal of mechanical property of fused deposition modeling processed parts. Materials \& Design 31(1): 287-295.

25. Thrimurthulu K, Pandey P, Venkata Reddy N (2004) Optimum part deposition orientation in fused deposition modeling. International Journal Of Machine Tools And Manufacture 44(6): 585-594.

26. Rivera ML, Moukperian M, Ashbrook D, Mankoff J, Hudson SE (2017) Stretching the bounds of 3D printing with embedded textiles. In Proceedings of the 2017 CHI Conference on Human Factors in Computing Systems 497-508.

27. Sculpteo. Filament for FDM 3D Printers.

28. SD3D Printing. Reprap $\mathrm{x} 400$ | German reprap x400 preis | 3d printing on demand.

29. Stratasys. What is FDM? Fused Deposition Modeling Technology for 3D Printing | Stratasys.

30. Valtas A, Sun D (2016) 3D printing for garments production: an exploratory study. Journal of Fashion Technology \& Textile Engineering 4(3): $1-4$. 